\title{
EchoGéo
}

$37 \mid 2016$

Femmes et migrations : celles qui restent

\section{Mobilités et immobilités des femmes qui " restent » dans la vallée du Rio Negro (Nicaragua)}

Anaïs Trousselle

\section{OpenEdition}

12 Journals

Electronic version

URL: https://journals.openedition.org/echogeo/14714

DOI: 10.4000/echogeo.14714

ISSN: 1963-1197

Publisher

Pôle de recherche pour l'organisation et la diffusion de l'information géographique (CNRS UMR 8586)

\section{Electronic reference}

Anaïs Trousselle, "Mobilités et immobilités des femmes qui « restent » dans la vallée du Rio Negro (Nicaragua)", EchoGéo [Online], 37 | 2016, Online since 07 October 2016, connection on 11 August 2021. URL: http://journals.openedition.org/echogeo/14714 ; DOI: https://doi.org/10.4000/echogeo. 14714

This text was automatically generated on 11 August 2021

EchoGéo est mis à disposition selon les termes de la licence Creative Commons Attribution - Pas d'Utilisation Commerciale - Pas de Modification 4.0 International (CC BY-NC-ND) 


\title{
Mobilités et immobilités des femmes qui « restent » dans la vallée du Rio Negro (Nicaragua)
}

\author{
Anaïs Trousselle
}

\section{Introduction}

Deux postures contradictoires polarisent la littérature quant à la place et au rôle que tiennent les femmes "qui restent " ${ }^{1}$, c'est-à-dire de celles qui ne migrent pas. Tout d'abord, certains auteurs soutiennent que les femmes sont passives dans le processus migratoire. La migration ne transformerait pas les structures familiales, notamment patriarcales. Elle aurait également pour conséquence d'augmenter la dépendance économique des femmes envers les hommes. En effet, la migration couplée à l'envoi de remises, conduirait à une réduction de l'implication dans les activités productives par les non-migrantes. De plus, si la migration produit certains changements de la distribution genrée des rôles, ceux-ci ne semblent pas toujours positifs. Parfois même, ils renforcent les inégalités de genre. Enfin, il est avancé que si changements il y a, ceux-ci s'estompent dès le retour des époux notamment (Mahler, 1999; Pessar, 2005 ; Taylor et al., 2006).

2 À l'inverse, d'autres auteurs démontrent que la migration opère des changements dans les rapports de pouvoir au sein de la famille et influence les divisions sexuelles du travail. Il est notamment mis en évidence l'augmentation des capacités de prises de décisions dont jouissent les femmes, qui renvoie à des formes d'autonomie suite au départ des hommes (Levitt, 1998 ; Kanaiaupuni, 1995).

Ce débat s'est construit sur la base de travaux empiriques qui témoignent de la diversité des situations et de l'importance de chaque contexte spatial et temporel. Il semble alors intéressant de se demander quels sont les facteurs clés qui modèrent cette relation entre femmes " qui restent » et migration des conjoints ou d'autres membres de la famille. Nous nous référons alors aux travaux d'Oso et Catarino (1997) concernant 
l'impact de la migration sur la possibilité des femmes de devenir chefs de ménage à la suite de la migration de leurs maris. Ces auteurs mettent en évidence quatre facteurs, à savoir : 1) la place des femmes dans le système de production ; 2) la structure familiale et leur position dans celle-ci ; 3) leur âge et leur position dans le cycle de vie ; 4) l'histoire migratoire des communautés à l'origine. Nous proposons ainsi de caractériser les réalités du quotidien de ces femmes «qui restent» dans le lieu d'origine ${ }^{2}$ en nous inspirant de cette littérature. Plus précisément, il s'agit de dépasser ces deux postures dichotomiques, en analysant la diversité des formes du rester, lesquelles impliquent souvent la mobilité non résidentielle ${ }^{3}$ de ces femmes. Nous proposons de mettre en particulier l'accent sur les éléments analytiques qui témoignent d'une articulation entre « ceux qui partent » et « ceux qui restent », articulation indispensable pour que la migration puisse se réaliser. Nous chercherons à répondre aux questions suivantes : en quoi la mobilité de certains membres de la famille influence-t-elle les projets de ces femmes "qui restent"? Quels rôles sont attribués à ces femmes en l'absence d'une partie de la famille? Quels changements sont à l'œuvre dans les pratiques et activités du quotidien de ces femmes? Quelles stratégies développent-elles alors pour se maintenir dans leur lieu d'origine?

4 Nous souhaitons contribuer en somme à une "géographie de l'absence » (Cortes, 2004; Poinard, 1991), c'est à dire décrypter la manière dont les femmes non migrantes vivent au quotidien les effets de l'absence. La démarche consiste, d'une part, à considérer la famille comme unité sociale d'analyse, au sein de laquelle nous repositionnons le rôle des femmes au regard de la migration des autres membres, quelle que soit leurs destinations à l'étranger ou au sein du pays. Il s'agit, d'autre part, d'analyser leurs manières de rester au travers du prisme de leur engagement dans les activités. Nous recourons ainsi au concept de Système Familial Multi-localisé (SFM) (Fréguin et al., 2015), lequel nous permet d'apprécier la capacité des familles à recourir à la dispersion et à la circulation pour capter des ressources humaines, sociales et économiques en vue d'assurer leurs moyens d'existence. Ce concept s'inscrit dans la continuité de travaux en géographie qui mettent en évidence la création de dispositifs réticulaires basés sur la mobilité et la dispersion spatiale des individus, lesquels sont liés aux autres par la production de circulations (matérielles et immatérielles). Ces dispositifs socio-spatiaux, prenant la configuration d'économies familiales en archipel (Quesnel et Del Rey, 2005), supposent le maintien de liens entre les migrants et leurs familles à l'origine tel qu'ont $\mathrm{pu}$ le mettre en évidence les approches transnationales (Glick Schiller et al., 1992) ou celles mobilisant la notion de circulation migratoire (Ma Mung et al., 1998). En tant que forme sociale d'organisation à distance, le SFM suppose alors 1) d'identifier le dispositif de multi-localisation familiale des membres d'une famille, à savoir l'ensemble des lieux de résidence et d'activité des individus ; 2) de comprendre leurs pratiques mobilitaires et leurs implications dans les différentes activités; 3) de caractériser les liens maintenus à travers la production de circulations entre les différents lieux de la dispersion familiale.

5 Dans une première partie, nous présentons le contexte géographique d'étude, à savoir une région du nord du Nicaragua (vallée du Rio Negro, département de Chinandega) où le phénomène migratoire est une composante ancienne et structurante des dynamiques à l'œuvre, tout comme l'agriculture est un moteur des activités locales, même si des recompositions liées au développement économique de la région sont également influentes. Dans un deuxième temps, nous exposons la méthodologie d'enquête mise en œuvre lors d'un travail de terrain réalisé en plusieurs étapes ${ }^{4}$ dans six communes 
rurales. Nous présentons, en troisième lieu, trois histoires de vie qui rendent compte des situations auxquelles font face ces femmes confrontées à la migration de membres de leur famille. Elles révèlent alors certaines stratégies pour se maintenir au quotidien dans leur lieu d'origine, tout en recourant à d'autres formes de mobilités. Ces études de cas nous permettent, dans un dernier temps, de faire ressortir les facteurs d'influence qui caractérisent et différencient les formes du rester au nord du département de Chinandega et de comprendre le sens que prend l'absence pour ces femmes «qui restent ».

\section{Contexte de la région d'étude}

6 La vallée du Rio Negro située au nord du Nicaragua, à la frontière avec le Honduras dans le corridor sec mésoaméricain (illustrations 1 et 2), est considérée, selon les statistiques nationales, comme un espace à dominante rurale. C'est une zone en position d'interface, de par sa localisation entre les plaines fertiles du Pacifique et les flancs des montagnes qui traversent le Nicaragua du nord au sud, mais également de par sa proximité avec des pôles frontaliers et urbains. Cette situation explique que l'agriculture ait toujours été attractive pour les habitants de cette région, au même titre qu'une diversité d'activités non agricoles, pour la plupart impliquant une mobilité spatiale aux échelles régionales, nationales ou internationales.

Illustration 1- Localisation de la zone d'étude

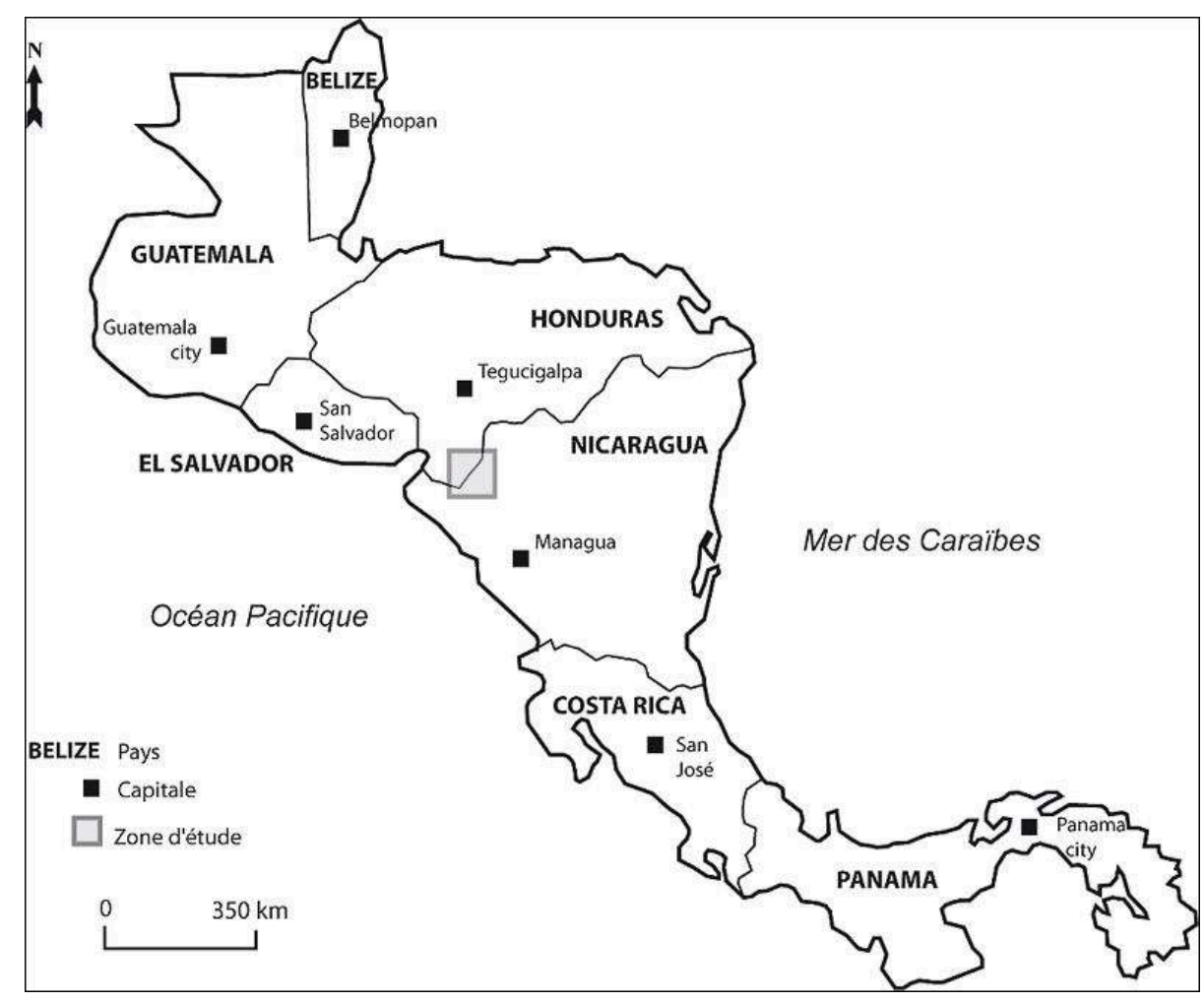

Source : A. Trousselle. 
Illustration 2 - Localisation des communautés d'origine au sein de la zone d'étude

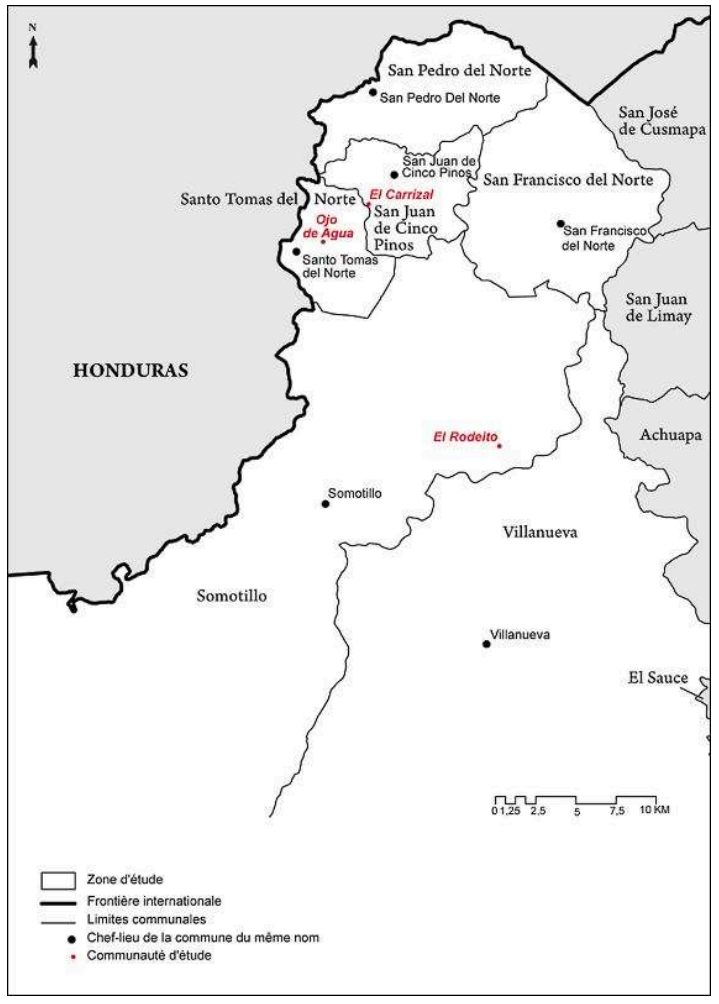

Source : A. Trousselle.

7 Au Nicaragua, la question agraire a souvent été au cœur des tensions politiques, économiques ou encore sociales, entrainant ainsi des recompositions récurrentes des formes de production agricoles et des activités non agricoles (Maldidier et Marchetti, 1996) dont la mobilité constitue l'un des principaux indicateurs.

$8 \mathrm{Au}$ cours du XXe siècle, la vallée du Rio Negro croît par le dynamisme que crée la frontière. L'arrivée de populations honduriennes dotées de moyens de production (force de travail, matériel et introduction de l'élevage bovin) ou encore le commerce transfrontalier, généré par l'existence de différents marchés ruraux et urbains, ont largement contribué à ce dynamisme.

En amont et durant la dictature des Somozas (1935-1979), la cristallisation du problème de l'accès à la terre se traduit en particulier par l'avancée de la frontière agricole. Celleci se réalise du Pacifique vers l'intérieur des terres, jusqu'à atteindre le nord du département de Chinandega qui devient alors une zone de refuge. La décennie 1980 marque un tournant dans l'histoire migratoire du pays, suite au triomphe de la révolution sandiniste en 1979. Deux grands couloirs migratoires se forment ou se réaffirment, l'un vers les États-Unis et l'autre vers le Costa Rica auxquels s'ajoutent des migrations internes liées aux conflits politiques et armés qui agitent le pays ${ }^{5}$. La décennie 1990 constitue également un tournant dans la trajectoire migratoire du pays avec l'accroissement des mobilités de travail ${ }^{6}$. Sur la période $1995-1999,59 \%$ des migrants à l'extérieur du pays sont au Costa Rica et $29 \%$ aux États-Unis (OIM, 2013). Un nouvel élan est donné au début des années 2000 avec près de $10 \%$ de la population nicaraguayenne à l'extérieur $\mathrm{du}$ pays et le développement de migrations intrarégionales $^{7}$ (vers le Salvador, Panama, Honduras) et outre atlantique (Espagne) 
(Baumester et al., 2008). L'espace mobilitaire nicaraguayen contemporain embrasse donc les échelles à la fois nationales, régionales et internationales (Fréguin et al., 2015).

Selon les données de l'OIM (2013), les femmes représentent 47,3\% de l'ensemble des migrants internationaux résidant à l'étranger. Elles sont cependant majoritaires au Costa Rica et aux États-Unis (respectivement 54,7\%,53,5\%), et plus encore au Panama (59,3\%) et surtout en Espagne (76\%). Concernant les autres formes de mobilités, peu d'études empiriques sont disponibles. Quelques éclairages sont apportés par Prunier (2013) et Winters (2014), soulignant l'importance d'analyser le rôle de ces femmes qui restent. Selon ces auteures, la capacité des femmes à s'extraire de la sphère domestique par la mobilité et l'engagement dans des activités rémunératrices dépend de leur organisation familiale.

11 La structure de la famille nicaraguayenne correspond à un mélange du modèle indigène et colonial qui se traduit par la superposition entre un modèle de famille monogame, légitimé en apparence mais également juridiquement, éthiquement et religieusement, et un modèle de relations polygames dans la pratique quotidienne (Fernandez Poncela, 1999). Au sein de cette structure type, surgissent alors deux unités familiales principales : la famille étendue qui regroupe des membres sur trois générations et la famille nucléaire au sein de laquelle on retrouve fréquemment la famille monoparentale, dont le chef du foyer est généralement une femme (ibid.). Dans ce contexte, les rôles confiés aux femmes sont nombreux pour assurer le fonctionnement de ces unités familiales. Elles sont en charge des activités domestiques, et plus largement de la subsistance matérielle de la famille. Elles assurent également la sécurité et le maintien des relations entre les membres de la famille. Ainsi, elles sont au service de la famille et en sont ses principales responsables et protectrices (ibid.).

La période postrévolutionnaire (années 1980), associée à de nombreux changements sociétaux, a incité à de nouvelles recherches sur la famille nicaraguayenne, ses dynamiques et ses membres. Ces analyses mettent en avant une reformulation des modèles traditionnels en termes de constitution et fonctionnement des unités de reproduction, production, accumulation, consommation, socialisation et pouvoir. Elles mettent en lumière notamment la diversification des stratégies de reproduction avec l'apparition de nouvelles composantes dont la migration interne et internationale (Jimenez, 2015). Ces changements se produisent au sein des familles, avec le constat de foyers bien plus mobiles basés sur la mise en mouvement continu de ses membres (Fauné, 1984). Ces changements sont caractérisés par des alternances entre des temps de permanence dans le lieu d'origine et des temps de mouvement dans l'espace à la fois rural, urbain, national et international. Ces recompositions ont des influences directes sur le rôle des femmes, qui sont elles-mêmes porteuses de ces dynamiques. Elles sont alors en charge de gérer ces nouvelles stratégies familiales en tant qu'organisatrices et administratrices du foyer se reconfigurant au rythme des migrations et ré-émigrations de ses membres. Elles sont donc celles qui développent et entretiennent tout un réseau social afin de surmonter les périodes de tension au sein de l'économie et de la vie des familles.

13 Dans la continuité de ces travaux, et au-delà des stratégies sociales que développent ces femmes, il est intéressant de comprendre les modalités de leur engagement dans les activités qui animent la zone d'étude. Outre les activités domestiques, elles sont mobilisées dans un certain nombre de tâches relatives aux activités agricoles mais aussi dans des activités de petits commerces. Elles participent également des 
reconfigurations récentes de la région avec l'émergence des activités minières (transformation artisanale confiée aux femmes) et de l'élevage de crevettes.

\section{Éléments de méthode : récits de vie et enquêtes multi- situées}

Issues d'une méthodologie de type qualitative, les données ont été collectées par entretiens et récits de vie visant tout d'abord à identifier les configurations sociospatiales $^{8}$ des familles et la localisation de leurs membres au moment de l'enquête. Elles ont permis ensuite de reconstituer les trajectoires de chacun des membres de la famille. L'objectif était de saisir les liens qu'entretiennent ceux qui restent et ceux qui partent, que ce soit en termes de relations sociales, de circulations matérielles et immatérielles ou d'organisation des activités.

Les récits de vie révèlent des témoignages relatifs à une même situation sociale, marquée ici par le départ en migration d'un proche ${ }^{9}$ (Bertaux, 2010). Répondant à une démarche multi-située, les entretiens ont été menés dans le lieu d'origine mais également dans certains lieux de destination afin de capter directement les histoires de vie des absents ${ }^{10}$. Nous avons également mis en place un suivi mensuel ${ }^{11}$ durant un an de certaines familles. L'objectif de ces suivis était de saisir les marqueurs de la mobilité et de l'absence (nouveaux départs, retours, appels téléphoniques ou internet, circulations matérielles, recherches de financements, remboursements et négociations des dettes, etc.). Cette méthode nous permet de sortir de la singularité de chaque histoire pour identifier des composantes fortes qui caractérisent le fait migratoire, et notamment les relations d'interdépendance entre ceux qui restent et ceux qui partent.

De ces entretiens et suivis de familles, nous proposons d'extraire trois études de cas de femmes restées dans le lieu d'origine. Leurs trajectoires témoignent de la diversité des situations à la fois familiales (statut dans les configurations familiales, cycle de vie de la famille), locales (activités réalisées et ressources disponibles) et mobilitaires (expériences et formes de mobilités au moment de l'enquête), situations qui façonnent les articulations entre partir et rester pensées à l'échelle familiale. Nous verrons plus loin que ces études de cas renvoient à un certain degré de représentativité des formes du rester identifiées au niveau de l'ensemble notre échantillon.

17 Notre échantillon ${ }^{12}$ se compose de 283 individus dont 136 femmes (48\%) et qui renvoient à 36 groupes familiaux ${ }^{13}$. Parmi ces femmes, $68 \%$ peuvent être considérées comme ayant une expérience de mobilité (expérience passée ou actuelle, avec ou sans changement de résidence). Au moment de l'enquête, 33 \% des femmes de l'échantillon étaient mobiles : $24 \%$ étaient en mobilité résidentielle dans des pôles urbains du pays (Chinandega, Managua), au Costa Rica, Honduras, en Espagne ou aux États-Unis, tandis que $10 \%$ recouraient à des mobilités non résidentielles de courte durée, vers les pôles urbains du pays (Chinandega, León), le Honduras, le Salvador et le Costa Rica. 
Tableau 1 - La mobilité au sein de l'échantillon

\begin{tabular}{|c|c|c|c|c|c|c|}
\hline & $\begin{array}{l}\text { Exp } \\
\text { mo } \\
\text { ou }\end{array}$ & $\begin{array}{r}\text { de } \\
\text { assées }\end{array}$ & $\begin{array}{l}\text { Expé } \\
\text { actu } \\
\text { mob } \\
\text { résid }\end{array}$ & de & $\begin{array}{l}\text { Expé } \\
\text { de } \\
\text { résid }\end{array}$ & $\begin{array}{l}\text { actuelles } \\
\text { és non }\end{array}$ \\
\hline $\begin{array}{l}\text { Femmes concernées par } \\
\text { l'étude }: \mathbf{n}=\mathbf{1 3 6}\end{array}$ & 92 & $68 \%$ & 31 & $23 \%$ & $14^{*}$ & $10 \%$ \\
\hline $\begin{array}{l}\text { Femmes résidant dans leur } \\
\text { lieu d'origine (au sein de la } \\
\text { zone d'étude) }: \mathbf{n}=\mathbf{9 7}\end{array}$ & 62 & $64 \%$ & & & 14 & $14 \%$ \\
\hline $\begin{array}{l}\text { Femmes « qui restent » dans } \\
\text { leur lieu d'origine (au sein de } \\
\text { la zone d'étude) }: \mathbf{n}=\mathbf{3 1} \mathbf{1}^{* *}\end{array}$ & 24 & $77 \%$ & & & 8 & $26 \%$ \\
\hline
\end{tabular}

${ }^{*}$ Ce chiffre est le même que celui de la ligne du dessous. Cela s'explique notamment par des difficultés méthodologiques à capter des mobilités non résidentielles mises en œuvre par des individus situés en dehors de la zone d'étude et que nous n'avons pas pu enquêter directement dans le lieu de destination.

**Ces femmes « qui restent » sont âgées de 16 à 69 ans avec un âge médian de 38 ans. 13 sont concernées par le départ d'un membre de leur foyer en tant que conjointes, 12 en tant que mères, 5 en tant que sœurs et 1 en tant que conjointe et mère à la fois.

Sur la totalité des femmes renseignées par les enquêtes, 97 résident dans leur lieu d'origine au moment de l'enquête (71\%) et 31 sont considérées comme les femmes " qui restent » puisqu'ayant au moins un membre de leur foyer est parti en migration (23\%). Parmi elles, $77 \%$ ont au moins une expérience passée ou actuelle de mobilités et, au moment de l'enquête, $24 \%$ de ces femmes qui restent pratiquent des mobilités non résidentielles de proximité et de courtes durées ${ }^{14}$. 3 femmes ont été chargées de s'occuper de leurs petits-enfants suite au départ d'un ou des parents. Malgré cette faible proportion de femmes en garde des enfants de migrants recensée par nos enquêtes, cette situation est pourtant fréquente dans la zone d'étude, comme dans d'autres parties du monde ${ }^{15}$ (Parreñas, 2005).

\section{Les formes de présence de la mobilité dans les histoires de vie}

Dans cette partie, nous proposons de mobiliser trois histoires de vie de femmes « qui restent» dans l'espace d'origine. Leur relation à la migration dépend tout particulièrement de leurs situations familiales et de la temporalité des migrations par rapport à leur cycle de vie. Le tableau 2 rend compte des principales caractéristiques de la composition de leur groupe familial et de l'incidence des mobilités. 
Tableau 2 - Composition des familles étudiées

\begin{tabular}{|c|c|c|c|c|c|c|c|c|c|c|}
\hline & \multirow[t]{2}{*}{ Âge } & \multirow[t]{2}{*}{$\begin{array}{l}\text { Génération } \\
\text { de } \\
\text { référence }\end{array}$} & \multirow[t]{2}{*}{$\begin{array}{l}\text { Nombre } \\
\text { d'individus } \\
\text { composant } \\
\text { le foyer }\end{array}$} & \multicolumn{2}{|c|}{$\begin{array}{l}\text { Nombre } \\
\text { d'individus } \\
\text { du foyer en } \\
\text { mobilité }\end{array}$} & \multirow[t]{2}{*}{$\begin{array}{l}\text { Nombre } \\
\text { d'individus } \\
\text { composant le } \\
\text { groupe } \\
\text { familial }\end{array}$} & \multicolumn{2}{|c|}{$\begin{array}{l}\text { Nombre } \\
\text { d'individus } \\
\text { du GF en } \\
\text { mobilité }\end{array}$} & \multicolumn{2}{|c|}{$\begin{array}{l}\text { Nombre } \\
\text { d'individus } \\
\text { du GF avec } \\
\text { une } \\
\text { expérience de } \\
\text { mobilité }\end{array}$} \\
\hline & & & & MR & MNR & & MR & MNR & MR & MNR \\
\hline Ana & 40 & G2 & 8 & 1 & 2 & 12 & 3 & 2 & 9 & 7 \\
\hline Clara & 32 & G3 & 4 & 0 & 1 & 13 & 6 & 1 & 8 & 3 \\
\hline Juana & 32 & G3 & 3 & 0 & 2 & 27 & 5 & 7 & 11 & 7 \\
\hline
\end{tabular}

MR : Mobilité résidentielle ; MNR : Mobilité non résidentielle. concernée par la migration en tant que conjointe, mère et grand-mère. Ses enfants et enfants de son conjoint ont de 16 à 31 ans et ses petits-enfants, qui constituent la $4^{\mathrm{e}}$ génération, ont de 1 à 15 ans. Les membres de sa famille sont dispersés au Costa Rica (avec plusieurs expériences migratoires dans ce pays) et en Espagne. La famille possède, en plus de leur maison, un patrimoine foncier (environ 2,5 hectares de terres agricoles et 0,5 hectare de forêt) ainsi qu'un puits sur une de leurs parcelles.

Clara (32 ans, originaire d'El Rodeito, municipe de Somotillo) a fondé son propre foyer et est concernée par la migration saisonnière en tant que conjointe. Clara est un

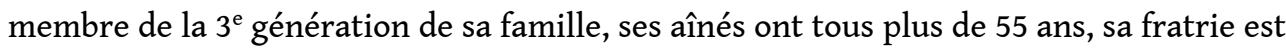
âgée de 30 et 34 ans et la $4^{\mathrm{e}}$ génération a de 3 à 12 ans. Les membres de famille migrent principalement au Costa Rica, où elle s'est déjà elle-même rendue dans le cadre de mobilités résidentielles. Elle et son conjoint possèdent en propriété leur propre maison, une moto, une paire de bœufs et un araire.

Juana (32 ans, vivant à Ojo de Agua, municipe de Santo Tomas del Norte) a fondé son propre foyer. Elle est également concernée par la migration de son conjoint aujourd'hui

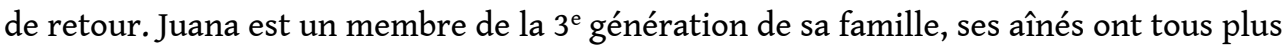

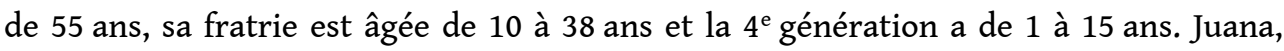
dont les expériences de mobilité se limitent aux bourgs proches, a une partie de sa famille qui réside aux États-Unis (La Nouvelle-Orléans). Elle et son conjoint possèdent en propriété leur maison ainsi qu'une autre qu'ils louent à Somotillo, ils disposent de 2 camions de transport de matériel et d'une moto acquis avec l'argent de la migration internationale du conjoint.

\section{Trajectoire d'Ana: de la migration internationale à l'immobilité dans le lieu d'origine}

Ana est âgée de 18 ans lorsqu'elle se met en couple. Avant d'être de "celles qui restent", elle a d'abord accompagné son mari dans ses migrations. En 1989, ils s'installent, avec les deux enfants de son conjoint, dans une communauté des plaines du 
Pacifique. En 1994, suite à la perte de leurs récoltes, ils décident alors de partir pour le Costa Rica. Ils resteront huit ans à Orotina où ils sont employés, Ana compris, comme travailleurs agricoles. En 2002, la famille rentre et fait construire une maison au Nicaragua dans leur lieu d'origine, El Carrizal. Ana y habite jusqu'en 2005, année à partir de laquelle elle rejoint son époux et un des enfants partis depuis 2003 pour s'installer dans une exploitation caféière au Costa Rica et y travailler comme cuisinière. En 2011, la fille ainée de son conjoint part en Espagne afin de travailler et d'épargner. La famille s'organise donc autour de ce nouveau projet migratoire qui conduit Ana à retourner au Nicaragua, avec ses enfants et les enfants de sa belle-fille et de son époux, afin de s'en occuper.

Ana entame ainsi une période "d'immobilité " qu'elle vivait encore au moment des enquêtes. Avec le départ de sa fille et l'éloignement de son mari (resté au Costa Rica), Ana devient chef d'un foyer composé uniquement d'enfants et petits-enfants et cesse toute activité autre que domestique. Il est convenu que les parents de ses petits-enfants se chargent d'assumer financièrement les cinq enfants et d'aider Ana également afin d'assurer les dépenses du quotidien.

Dans cette nouvelle configuration familiale, le moindre déplacement d'Ana implique une organisation avec son fils cadet car les petits-enfants nécessitent une prise en charge permanente. Ana vit au rythme des allers et retours de son mari, ainsi que de ceux de l'un de ses fils parti étudier à León. Elle dépend aussi des envois de biens matériels (argent, nourriture, fournitures scolaires) provenant d'Espagne et du Costa Rica qu'elle mobilise pour l'avancée des projets individuels et collectifs de la famille, même si leurs ressources, foncières notamment, ne peuvent être exploitées du fait de l'absence des adultes de la famille. En décembre 2014, un de ses petits-enfants ainsi qu'elle-même tombent malade. Elle en avertit alors les parents pour qu'ils prennent en charge les frais relatifs aux soins de son petit-fils. Préoccupée par la scolarité des enfants et leur santé, et limitée dans ses possibilités de déplacement en ville pour des consultations, elle passe sous silence sa maladie auprès de ses proches. C'est son fils cadet qui la force, au retour de son mari, à révéler sa situation de santé. Cette situation conduit la famille à se réorganiser afin de décharger Ana de ses tâches quotidiennes qu'elle n'est plus en mesure d'assumer. Pour Ana, ajouter ses problèmes de santé aux difficultés quotidiennes des mobilités de ses proches lui fait éprouver un sentiment de culpabilité. La famille finance donc le salaire d'une femme de ménage, mais Ana souligne que cela ne soulage pas sa solitude de devoir affronter ses problèmes de santé seule en l'absence des autres, et notamment de son mari. Ce dernier fait le maximum pour être à ses côtés lors de ses retours, mais la charge importante de travail qu'implique la période de récolte du café au Costa Rica contraint ses possibilités.

En janvier 2015, Ana, toujours malade, doit annoncer à sa belle-fille en Espagne que sa fille âgée de 15 ans, est partie s'installer chez son conjoint arrêtant ainsi l'école secondaire. Les deux femmes expriment leur impuissance face à cette situation préoccupante. Quelques mois plus tard, la mère de cette dernière annonce à Ana qu'elle a rencontré quelqu'un en Espagne. Amoureuse, elle souhaite donc se séparer de son conjoint et rester plus longtemps en Espagne, ne sachant donc pas quand elle rentrera. En parallèle, son conjoint, toujours dans l'attente de son retour, s'apprête à prendre la route vers les États-Unis afin d'aider financièrement sa conjointe en Espagne et d'avancer son retour. Ana ne sachant plus sur qui compter se tourne vers le père de ses petits-enfants pour lui demander comment il compte gérer les mois à venir. Lors d'un 
entretien succédant ces nouvelles, Ana regarde le tas de briques de construction entassées dans le jardin, qu'elle s'était chargée d'acheter, destinées à la construction de la maison de sa belle-fille et son conjoint en me disant :

«Quel sens cela a maintenant? Sans le retour de Karen, cela n'en n'a plus aucun » (entretien, avril 2015).

Les reconfigurations des projets individuels viennent remettre en question la situation et le rôle d'Ana au sein de ce dispositif familial. Persuadée que la famille se réunirait prochainement dans leur lieu d'origine, elle avait ainsi accepté de renoncer à sa mobilité et à son travail. Avec la disparition d'une perspective de retour de sa bellefille, les perspectives d'Ana sont remises en question tout comme le sens de son attente. La trajectoire de la famille d'Ana (illustration 3) démontre une forte projection collective dans la communauté d'origine. La migration internationale permet d'acquérir des ressources foncières dans le lieu d'origine mais qui ne peuvent être exploitées (terres agricoles principalement), la main-d'œuvre familiale faisant défaut. Le dispositif de dispersion de cette famille, autrement ce système familial multilocalisé, s'accompagne d'un ancrage fort dans la communauté d'origine, permis par la présence d'Ana qui veille sur le patrimoine familial et prend en charge les plus jeunes membres de la famille. Néanmoins, le dispositif collectif est facilement fragilisé lorsque les projets individuels de ceux en mobilité changent, et les effets de ces changements impactent alors directement celle qui reste.

Illustration 3 - Trajectoire d'Ana et de sa famille

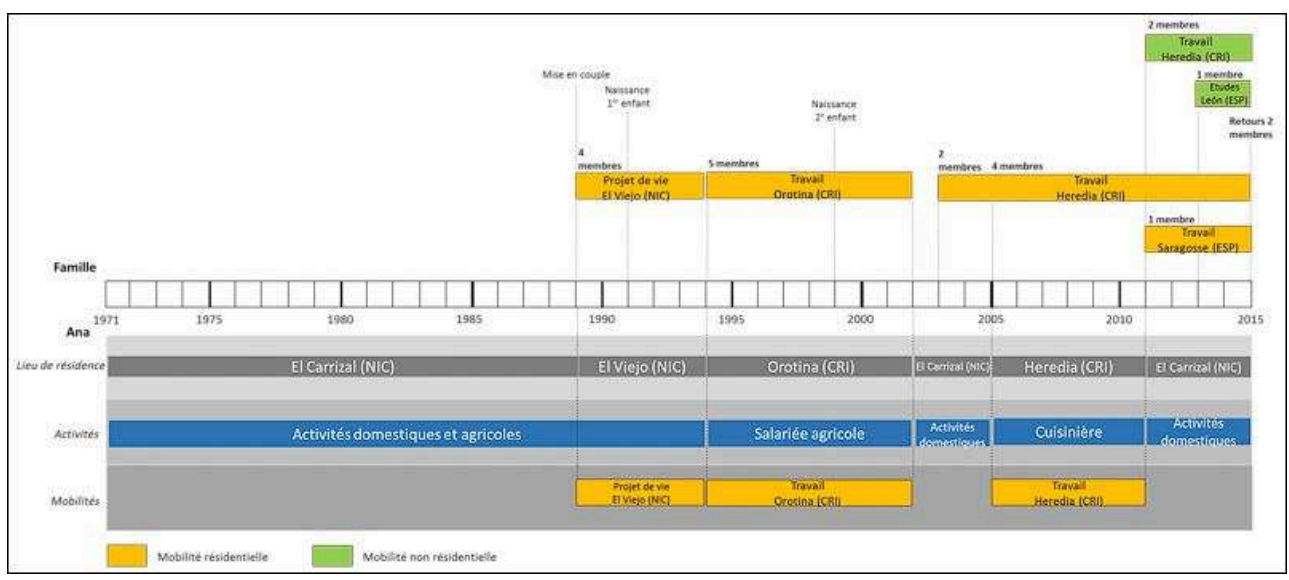

\section{Trajectoire de Clara : le recours à la mobilité de proximité pour affronter le quotidien en attendant le retour des autres}

Clara, originaire de la localité d'El Rodeito au Nicaragua, a 17 ans quand elle se met en couple en l'an 2000. À cette date, elle a deux expériences de mobilité, l'une résidentielle vers Chinandega, l'autre hebdomadaire vers Somotillo pour poursuivre sa scolarité. Le jeune couple s'installe chez la mère de Clara qu'ils appuient dans les activités domestiques et agricoles. En 2004, ils décident de partir avec leur fille pour San José (Costa Rica), où le conjoint de Clara s'est déjà rendu à plusieurs reprises, afin de travailler pour financer la construction de leur maison. Après quelques mois, Clara confie sa fille à la grand-mère et commence à travailler comme domestique. Son 
conjoint, quant à lui, travaille comme ouvrier dans la construction sur des chantiers dans tout le pays, ce qui suppose des mobilités hebdomadaires. En 2007, ils décident de rentrer à El Rodeito au Nicaragua et y établissent leur foyer. Dès 2008, son conjoint effectue des migrations saisonnières de février à mai (saison sèche) vers Santa Rosa de Lima (El Salvador), vers San José et en 2014 à Colón (Panama). Le reste du temps, il se consacre à l'agriculture et à des travaux de maçonnerie dans les communautés voisines mobilisant les compétences acquises au Costa Rica. Clara assure les activités domestiques ainsi que certaines tâches dans l'agriculture. Lors des périodes de migrations saisonnières de son conjoint, Clara exprime son sentiment d'être assignée à domicile. Pour la moindre mobilité ponctuelle, elle doit pouvoir faire appel à sur sa mère pour s'occuper de ses filles le temps de son absence. Pourtant, les bénéfices économiques des migrations saisonnières de son conjoint n'étant jamais garantis, elle se doit de renforcer certaines années ses mobilités non résidentielles afin d'assurer le quotidien. Avec une de ses amies de la communauté, elle se rend dans les communautés des alentours lors de rassemblements diverses afin de vendre de la nourriture. L'envoi d'argent par son mari peut tarder à venir et leur niveau d'endettement est très élevé.

Ainsi, les activités de Clara dépendent des modalités des migrations de son conjoint (durée, destinations, emplois, etc.) et des résultats des activités agricoles. En 2014, les migrations saisonnières de celui-ci n'ont permis ni de rembourser les prêts contractés pour financer le voyage, ni d'en tirer un quelconque gain financier. Il leur a donc fallu emprunter à nouveau pour financer les cycles de culture qui ont suivi. Mais cette même année, le couple a perdu toute sa récolte. Le couple a ainsi fait le choix d'emprunter une nouvelle fois début 2015 afin que le conjoint de Clara puisse partir en février et rembourser les importantes dettes accumulées. Pour Clara, cette situation est source d'inquiétudes qu'elle peine à dissimuler malgré une force de caractère reconnue au sein de sa communauté :

«Tout va bien, enfin je veux dire nous ne sommes pas malades et avons de quoi manger aujourd'hui...mais aussi, ça ne va pas, je suis préoccupée par toutes ces dettes que nous avons, parfois je me demande même si nous pourrons offrir à Gwendie un bel anniversaire pour ses $15 \mathrm{ans}^{17}$, surtout que Pedro m'a dit hier que ça lui coutait de plus en plus d'être loin » (entretien, avril 2015).

31 Clara, en plus de la "débrouille " au quotidien, des inquiétudes sur leur avenir et du réconfort à apporter à son conjoint, doit également ménager les réclamations de ceux à qui ils doivent de l'argent, à savoir des membres de la communauté et de leur famille. Rester quand son conjoint part, impose à Clara d'être la médiatrice auprès des membres de sa communauté et de sa famille pour gérer les tensions sociales que le remboursement tardif des dettes instille. Lorsque l'épargne de la migration finit par arriver, elle sacrifie les besoins de sa famille pour payer certaines créances. Elle commence toujours par rembourser les membres qui ne sont pas de sa famille, moins aptes à tolérer des retards trop importants. La souplesse de la famille, qui traduit un certain niveau de solidarité entre proches, a cependant un coût en termes d'obligations. L'un des frères de Clara résidant au Costa Rica, qui est aussi un des principaux supports financiers du couple, lui confie les différents travaux agricoles de ses cultures de sésame. Son conjoint rentre ainsi chaque saison, fournissant son travail en contrepartie des possibilités de prêts accordés par le frère. En cette phase d'endettement, son conjoint est d'autant plus sollicité, ce qui devient une véritable contrainte pour le 
couple qui doit aussi assurer ses propres cycles de culture en parallèle. Pour Clara cela implique notamment de préparer davantage de repas pour les travailleurs journaliers et de participer à différentes tâches agricoles.

La trajectoire de cette famille (illustration 4) montre une articulation entre activités locales et en migration où l'accumulation de ressources est difficile car fortement dépendante des contextes (marchés de l'emploi, caractéristiques de la saison agricole). La cohésion familiale apparaît alors à la fois comme un moyen d'éviter les situations extrêmes et comme une contrainte qui conditionne, dans une certaine mesure, les projets migratoires et les activités exercées. En l'occurrence, Clara est une figure centrale dans la gestion de ce dispositif socio-spatial.

Illustration 4 - Trajectoire de Clara et de sa famille

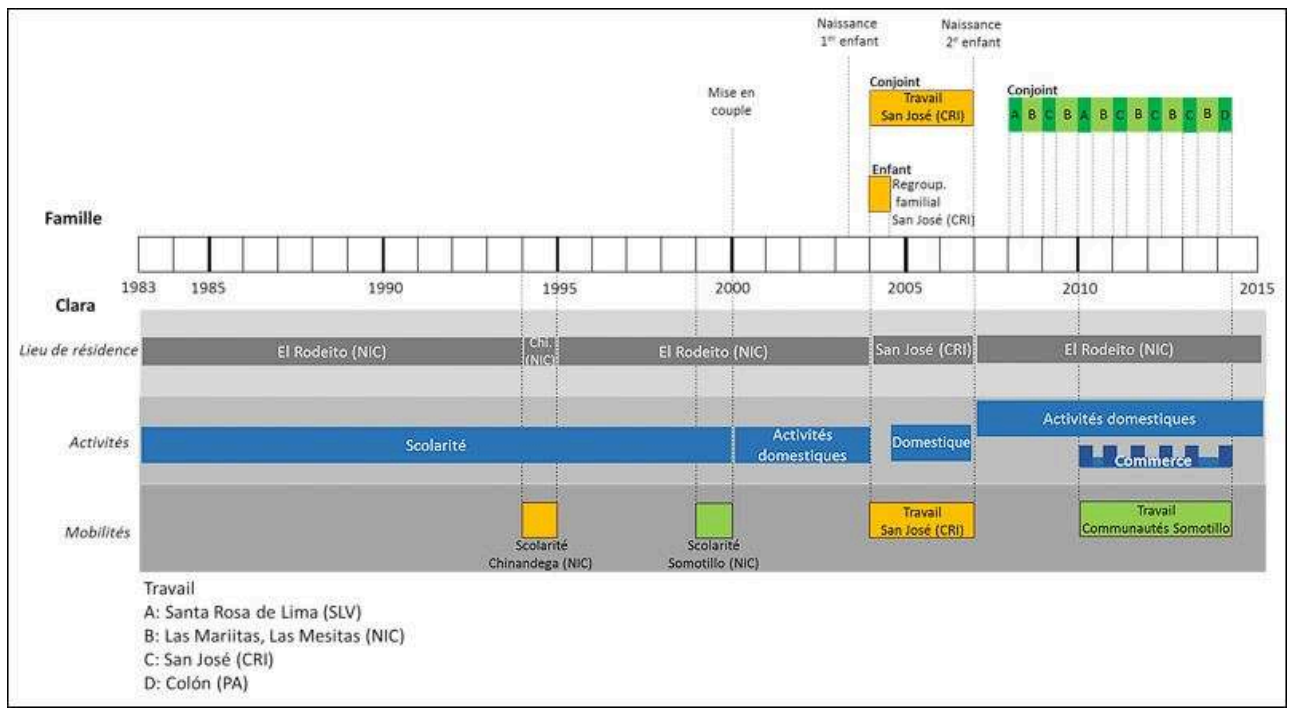

\section{Trajectoire de Juana : le renforcement des activités par la mobilité}

Juana, originaire d'Ojo de Agua, est issue d'une fratrie de onze enfants, avec un niveau élevé de cohésion familiale. Ses parents ont construit leur patrimoine et développé des activités rémunératrices grâce à leurs migrations saisonnières dans les plaines du Pacifique. Au fil des années, ils ont pu capitaliser dans leur communauté d'origine, Ojo de Agua. Ils sont maintenant à la tête de plusieurs hectares, d'une épicerie et d'un réseau de camionnettes de transport de matériel. Lorsqu'elle rencontre son conjoint en 2005, Juana s'engage avec une de ses sœurs dans une activité de vente de cosmétiques. Elles vont deux fois par mois s'approvisionner au Honduras et revendent leurs marchandises dans les communautés des alentours. À peine un an après son union, alors que le couple habite encore chez les parents de Juana, son mari part pour les États-Unis, après de multiples expériences au Costa Rica, au Honduras et au Salvador. Il espère ainsi épargner pour qu'ils puissent fonder leur propre foyer à son retour.

Juana devient ainsi une jeune épouse seule, avec pour garantie les promesses de son conjoint absent. Juana se rappelle :

«On ne se connaissait pas depuis si longtemps, comment savoir ce qu'il se passait là-bas surtout quand son homme n'exprime pas ses souffrances et ses 
faiblesses? Parfois je me disais que j'avais mis trop d'espoir dans ce projet de vie qui pouvait s'effondrer à tout moment » (entretien, juillet 2014). cependant avec le départ de sa sœur pour les États-Unis. En 2010, son mari lui confie l'organisation des premières étapes de la construction de leur maison, sûrement pour se rassurer mutuellement de l'avancée de leur projet de vie, précise Juana. Elle se retrouve donc dans une nouvelle maison en construction qui l'oblige à réduire ses mobilités pour garder la maison. Elle se rappelle que cette période de près de deux ans lui a coûté car elle a pris conscience qu'elle aimait travailler et ne pas être cantonnée aux activités domestiques. En 2012, son conjoint est de retour et l'aide à financer de nouvelles activités plus rémunératrices. Elle reprend ses ventes itinérantes mais avec des produits qui rapportent davantage. Juana vend désormais des appareils électroménagers achetés à Choluteca au Honduras. Elle revend également des vêtements d'occasion achetés à Chinandega ${ }^{18}$. Les économies de son conjoint ainsi que ses bénéfices permettent au couple d'acheter, entre autres, une moto et d'accroître son aire d'activité. Pour Juana, c'est à partir de ce moment que la migration précédente de son conjoint se révèle positive. Elle leur a permis de s'installer sans avoir besoin de repartir, dit-elle. En 2013, Juana donne naissance à leur premier enfant, ce qui réduit ses déplacements. Son conjoint, originaire du bourg de Somotillo développe des mobilités de travail diverses au sein de la zone d'étude. Grâce à sa famille restée très proche et pouvant prendre soin de son fils, Juana continue d'exercer ses activités à rythme réduit. C'est pour elle un accomplissement personnel. Depuis 2014, elle a développé une nouvelle activité de prêts alloués aux migrants saisonniers qui se rendent au Costa Rica et au Panama. Tout en étant "de celles qui restent», elle participe ainsi à la mise en mobilité d'autres membres de sa communauté. En 2014, profitant d'une opportunité de travail, son conjoint se rend trois mois au Salvador, Juana réduit donc son activité et sa mobilité. Elle n'exprime cette fois plus de souffrance, convaincue que ces mobilités contribuent au final à la prospérité de son foyer.

La trajectoire de ce couple (illustration 5) montre que la mise à disposition de ressources familiales leur a permis de consolider leurs propres projets et d'accumuler des ressources via la migration internationale. Les mobilités de Juana, comparées au cas précédent, renvoient davantage à un désir d'autonomie et d'accomplissement personnel plutôt qu'à la nécessité de trouver des revenus pour la subsistance quotidienne. 
Illustration 5 - Trajectoire de Juana et des membres de sa famille

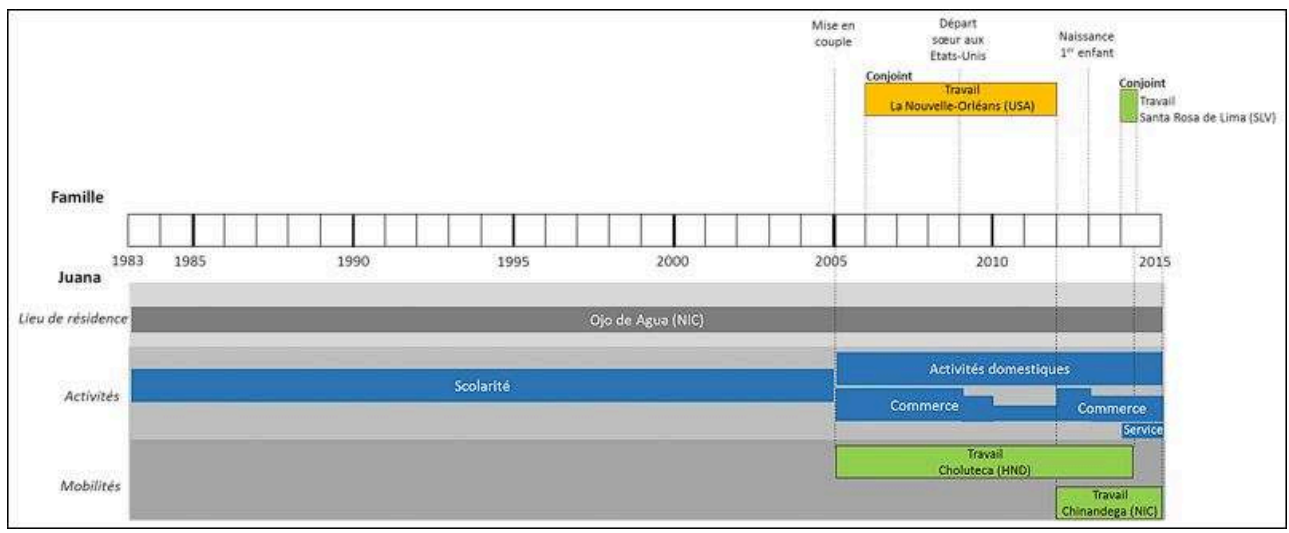

\section{Caractériser « le rester » dans le lieu d'origine}

Ces trois expériences singulières $d u$ "rester» dans le nord du département de Chinandega mettent en lumière des éléments qui nous semblent centraux dans la caractérisation du vécu des femmes qui font l'expérience «indirecte» de la migration. Nous détaillons dans cette section trois de ces éléments qui renvoient à un certain degré de généralité au niveau de l'échantillon global : l'articulation entre la migration des uns et les mobilités ou immobilités du quotidien de celles " qui restent ", le rôle des femmes dans les circulations de biens matériels et immatériels, et les difficultés que véhicule l'absence et qu'engendre la migration pour ces femmes.

\section{Les mobilités et immobilités du quotidien de celles " qui restent » s'articulent aux migrations des autres}

Les histoires de vie nous révèlent que le fait de rester dans le lieu d'origine conduit parfois ces femmes à devenir immobiles ou bien à mettre en œuvre de mobilités de proximité et de courtes durées. Ces situations s'articulent aux autres formes de mobilités pratiquées par ces femmes et surtout par les membres de leur famille, révélant dans tous les cas des stratégies de maintien dans le lieu d'origine. Nous proposons une typologie qui révèle trois types d'articulation entre les différentes formes de mobilités, illustrant la complexité des rapports entre sédentarité et mobilité.

Tout d'abord, lorsque certains membres de leur foyer (voire de leur groupe familial) famille migrent, les femmes sont parfois amenées à interrompre leurs propres mobilités (résidentielles ou non) pour retourner ou se fixer dans le lieu d'origine. Cette situation témoigne généralement de la volonté de certains membres de la famille à investir davantage dans la communauté d'origine (accumulation de ressources, notamment foncières, ou acquisition de bétail et amélioration/construction de l'habitat). Dans la mesure où les mobilités féminines s'interrompent, nous parlons dans ce cas d'une articulation de type annulation. Six des 31 femmes de notre échantillon " qui restent » dans leur lieu d'origine sont concernées.

Lorsque ces femmes développent des mobilités non résidentielles, simultanément à la migration de travail d'autres membres de la famille, notamment à l'international, nous parlons d'une articulation de type superposition. Cela concerne 7 des femmes «qui 
restent ». Celles-ci ont en commun d'avoir à affronter le quotidien en attendant les retombées de migrations à plus long terme. Il s'agit généralement de jeunes individus qui viennent de se mettre en couple ou d'avoir des enfants, et qui démarrent la constitution d'un patrimoine familial, par exemple la construction ou l'achat d'une maison. Dans plusieurs cas, ces superpositions de mobilités correspondent à un regroupement familial temporaire, les femmes rejoignant pour quelques semaines leur conjoint dans le lieu de destination de manière à l'appuyer dans leur projet commun.

41 Lorsque les membres de la famille reviennent au lieu d'origine, après un temps de migration, et que les femmes qui étaient restées s'engagent alors dans des mobilités non résidentielles, nous parlons d'une articulation de type succession. Deux femmes sont concernées par cette situation. Ces mobilités non résidentielles, soutenues par les ressources de la migration internationale, permettent de capter de nouvelles ressources dans les espaces proches du lieu d'origine.

Enfin, la majorité des femmes qui restent (16 sur 31) n'était pas engagée dans une mobilité avant le départ d'un ou plusieurs membres de leur famille et ne l'a pas été au cours de ces mobilités familiales ${ }^{19}$. Les raisons de cette immobilité peuvent être diverses : 11 d'entre elles sont des femmes «qui restent» en tant que mère au foyer appartenant généralement aux générations 1 voire 2 au sein des familles. Elles ont un ancrage fort dans leur communauté d'origine. Leur subsistance repose sur des ressources locales (agriculture ou autre) et/ou provient des revenus de la migration de leurs enfants. Les 5 autres (parmi les 31) sont fortement impliquées dans des projets familiaux leur permettant de capter directement des ressources locales et migratoires. Trois sont notamment centrales dans la réalisation des projets des individus en mobilité (gestion des transferts d'argent et projets en cours dans le lieu d'origine).

Tableau 3 - Les différents types d'articulation entre celles qui restent et ceux/celles qui partent

\begin{tabular}{|c|c|c|c|c|}
\hline & «Annulation » & «Superposition» & «Succession» & Immobilité \\
\hline \begin{tabular}{llll} 
Femmes & "qui & \multicolumn{2}{c}{ restent» } \\
actuellement dans leur lieu \\
d'origine (au sein de la \\
d'étude)
\end{tabular} & 6 & 7 & 2 & 16 \\
\hline
\end{tabular}

43 La première forme d'articulation entre les différentes mobilités (annulation) surgit lorsque des membres de la famille partent en migration pour des durées conséquentes. Cette situation conduit parfois ces femmes « qui restent » à s'immobiliser dans leur lieu d'origine, mettant fin à leurs propres mobilités (résidentielles ou non). Cette annulation du projet mobilitaire ${ }^{20}$ va généralement de pair avec la fin d'un travail salarié ou la réduction de leur engagement dans des activités rémunératrices. Ces situations transforment au moins temporairement leurs rôles au sein de la famille, puisqu'elles sont renforcées dans leur rôle de gestionnaire du foyer avec la responsabilité d'assurer la satisfaction des besoins du quotidien. Leur participation à la production de revenus est ainsi diminuée et, on l'a vu, certaines d'entre elles se sentent assignées à domicile, notamment celles confrontées à la migration internationale au sein de leur famille. Cette situation dépend également de l'étape dans le cycle de vie familial. En effet, cela coïncide avec la phase d'installation dans le lieu d'origine et la 
construction de la maison ou avec encore l'arrivée du premier enfant. L'immobilité des femmes qui restent devient alors incontournable pour la mise en œuvre de la stratégie familiale.

La seconde forme d'articulation entre les différents types de mobilités (superposition) traduit des stratégies féminines qui visent à assurer le quotidien en attendant les retombées de migrations plus longues. Ces femmes maintiennent ou renforcent alors des mobilités de proximité pour développer de nouvelles activités rémunératrices, tout en assurant les activités habituelles (domestiques, agricoles, etc.).

Cette configuration renvoie au caractère risqué de la migration internationale avec, lors de sa mise en œuvre, une phase d'adaptation où le migrant a besoin de temps pour trouver un emploi, percevoir une rémunération dans des contextes où il est généralement vulnérable de par son statut de clandestin. Les retours vers le lieu d'origine et les envois d'argent peuvent donc tarder à arriver et être à tout moment réduits en fréquence et intensité (Trousselle, 2012).

Dans le lieu d'origine, il faut donc que les femmes "qui restent» composent en attendant, et les mobilités de proximité peuvent être un palliatif, à condition d'avoir les moyens de les mettre en œuvre et de pouvoir s'extraire de la sphère domestique. La force des liens familiaux est alors déterminante pour permettre à ces femmes de se mouvoir dans les espaces de proximité, d'autant plus qu'il s'agit généralement de jeunes individus qui préparent leur mise en couple ou l'arrivée d'enfants. Il faut alors pouvoir compter sur des membres de sa famille (la grand-mère est fortement mobilisée) pour garder les enfants (quand elles en ont déjà), préparer les repas, surveiller la maison, avancer de l'argent pour un ticket de bus ou pour acheter des ingrédients qui seront vendus transformés, etc.

47 La troisième forme d'articulation, celle de la succession, concerne les mobilités résidentielles généralement pratiquées par les conjoints ou les enfants et les mobilités non résidentielles des femmes qui restent (et parfois par les conjoints de retour). La mobilité résidentielle, lorsqu'elle aboutit au retour du migrant au sein de son foyer d'origine, correspond à de nouvelles ressources dans le lieu d'origine. Ces femmes se saisissent alors de l'épargne de la migration pour développer ou renforcer leurs mobilités non résidentielles afin de capter des opportunités d'activités et de ressources monétaires.

Cette situation renvoie à des femmes qui disposent d'un « capital mobilité » c'est-à-dire qu'elles ont des expériences de mobilités antérieures qui renforcent leur capacités à se déplacer. Ces femmes remobilisent ainsi des réseaux mais aussi des savoir-faire acquis pendant les périodes de mobilités précédentes et pendant la période du "rester ». En d'autres termes, la condition du rester est réversible: une fois que les capitaux sont disponibles, et la famille réorganisée suite au retour de certains membres de la famille, la femme peut reprendre ou entreprendre des activités qui impliquent sa propre mise en mobilité.

\section{Les circulations, témoins du rôle des femmes au sein du système familial multi-localisé}

Les circulations matérielles et immatérielles sont centrales dans les projets migratoires à plusieurs titres. D'une part, elles traduisent et (re)produisent la cohésion familiale. 
D'autre part, elles permettent de prendre la mesure des avancées des projets migratoires individuels et familiaux. Enfin, analyser l'usage qu'en font les femmes qui les réceptionnent permet de rendre compte des apports de la migration, notamment pour les communautés de la zone d'origine. Nous revenons sur les différents rôles confiés à ces femmes analysés au travers des circulations.

50 Tout d'abord, qu'elles soient conjointe, mère, grand-mère ou même sœur, ces femmes sont celles qui réceptionnent et redistribuent les biens, les capitaux et les informations qui proviennent d'ailleurs ${ }^{21}$. Elles assurent ainsi la cohésion familiale qui généralement est centrale dans leur discours.

51 Elles portent également la responsabilité de convertir ces circulations, en particulier les transferts d'argent, de manière à faire avancer les projets individuels et collectifs. Cela se traduit par l'achat de matières premières nécessaires à la construction d'une maison, l'achat de semences pour préparer le cycle de culture à venir, l'ouverture de compte en banque et le dépôt régulier de l'épargne des migrants internationaux, qui reviendront peut-être un jour des projets pleins la tête.

Lorsqu'elles sont chargées de rembourser les dettes, ces femmes deviennent également des médiatrices des pressions sociales. Elles doivent gérer les tensions intrafamiliales, de voisinage ou intracommunautaires en se déplaçant pour rencontrer les gens, communiquer sur les avancées des migrations des absents. À cela s'ajoute souvent la nécessité d'assumer des charges supplémentaires liées aux tâches domestiques quotidiennes ou aux activités agricoles (préparation des repas pour les travailleurs agricoles, etc.).

Ces femmes sont également les garantes du maintien du patrimoine familial. En effet, cette articulation entre ceux qui partent et ceux qui restent se traduit par l'activation ou la désactivation, sur des périodes plus ou moins longues, de certaines activités notamment agricoles. Lorsque certaines activités sont interrompues, il faut néanmoins maintenir les ressources nécessaires à leur réalisation. Ce rôle est alors confié aux femmes qui restent. Elles se chargent notamment d'entretenir la maison, les parcelles qu'elles soient ou non mises en culture ou encore le matériel agricole (puits, enclos, charrettes, etc.). Elles utilisent donc une partie de l'argent envoyé par les migrants à cette fin, en achetant des fournitures pour améliorer l'habitat, en employant ponctuellement des travailleurs agricoles pour réparer les clôtures ou nettoyer les parcelles, etc.

54 Enfin, ces femmes, parce qu'elles ont un certain degré d'ancrage dans le lieu d'origine, permettent de générer des mises en mobilité à l'échelle communautaire. Pour cela, elles convertissent certaines remises en argent disponibles pour le prêt à des candidats à la migration. De plus, elles diffusent les informations fournies par ceux déjà partis, concernant des opportunités d'emploi, développant ainsi des filières de recrutement au niveau communautaire.

55 Ces différents rôles, dont se dotent les femmes qui restent, témoignent d'une certaine capacité à assumer, en l'absence des conjoints notamment, de nouvelles fonctions qui traduisent souvent des processus d'émancipation et d'affirmation personnelle. Mais ces dynamiques s'accompagnent également de nombreuses pressions auxquelles elles doivent faire face. 


\section{Les réalités de l'absence et de l'attente qu'engendre la migration pour ces femmes « qui restent"} rôle de pilier que la famille leur attribue. À cela s'ajoute le contrôle social souvent accru exercé parfois par leur environnement social qui contraint d'autant plus leur quotidien. À ce sentiment d'isolement s'ajoute souvent celui d'une impuissance face à leur propre destin et celui de leurs proches. et d'inquiétude quant à leur couple, leur famille et leur devenir. Elle fait écho à des observations récurrentes dans la zone d'étude. Nombreuses sont celles qui indiquent souffrir de l'absence d'un conjoint ou d'enfants. Dans leurs maisons où sont généralement exposées les photographies de tous ceux et celles partis, aborder le sujet est toujours délicat. C'est le plus souvent par un rapprochement entre la situation de chercheuse - aussi concernée par un processus de mise à distance - et de celle de femme, de mère, ou encore de sœur, dont les proches sont loin, que la question est abordée. Nous retrouvons ici une marque classique des discours et des situations de souffrances qui sont caractérisés par une difficulté de mise en mots et qui transparaissent dans les silences, les regards, ou encore les non-dits (Vermot, 2015). Le confinement de la souffrance individuelle est en grande partie lié au rôle de pilier que ces femmes doivent assumer.

Il est souvent attendu de celles qui restent qu'elles soient à l'écoute et portent les difficultés que les migrants rencontrent au cours de leur trajectoire. Ce rôle de «soutien " conduit d'autant plus au confinement et au refoulement des souffrances qu'elles peuvent éprouver au regard de leur situation. En l'absence notamment des conjoints, ces femmes subissent aussi des nouvelles formes de contrôle social de la part de la communauté et de la belle-famille. En effet, la majorité des femmes enquêtées évoquent facilement ce sentiment d'être espionnées par leur environnement social même proche. Leurs déplacements sont surveillés, leurs fréquentations notées, les justifications à apporter nombreuses car généralement les conjoints sont mis au courant.

59 À cette solitude, s'ajoute un état d'impuissance qui est en décalage avec le rôle qui est assigné à celles qui restent. Les attentes qu'ont les membres des familles envers ces femmes «ressources » sont très élevées: prendre soin des enfants, s'assurer qu'ils poursuivent leurs études, garder la maison, surveiller les champs et les troupeaux ou encore réconforter ceux qui sont loin. Mais en pratique, ces femmes dépendent, dans une certaine mesure, des projets de vie des autres membres de la famille, de leurs ressources et de leur autorité. Comment faire face à un jeune qui décide d'arrêter l'école quand ce n'est pas le sien? Comment maintenir ses droits sur le puits familial face aux voisins quand les activités agricoles sont mises en suspens en attendant le retour des hommes? Comment assurer les travaux agricoles d'un prêteur quand on a ses propres parcelles à charge ? Les situations d'impossibilité sont multiples et variées. L'impuissance est d'autant plus conséquente que les difficultés sont souvent peu prises en compte par ceux qui sont absents qui doivent eux aussi faire face à d'autres obstacles dans les lieux de migration.

Les femmes "qui restent" incarnent de manière privilégiée ces contradictions entre rôles attendus et ressources disponibles. En effet, le facteur limitant est généralement 
humain car la force de travail familial et la main-d'œuvre disponible sont insuffisantes pour maintenir les systèmes de pluriactivité des familles. Les ressources locales ne peuvent donc pas toujours être exploitées pour produire des revenus nécessaires à la vie quotidienne des membres qui restent (via les récoltes, les produits agricoles transformés, etc.) et le besoin de liquidités pourvu par la migration devient donc fort. Ces contradictions s'expliquent par l'existence de formes de distance complexe aussi bien physique que de représentation qu'il est donc important de documenter; ce, afin de ne pas tomber dans des approches qui, soit inculpent les femmes de passivité dans le processus mobilitaire, soit valorise leur émancipation en faisant abstraction des contraintes associées. Cette étude tente justement de montrer qu'il existe une pluralité de processus produits par cette articulation entre celles qui restent et ceux qui partent. Il apparaît alors pertinent de considérer l'âge de ces femmes et leur position dans le cycle de vie, la structure familiale et leur position dans celle-ci, les activités exercées dans le système de production et les ressources à disposition afin de comprendre ces formes du rester.

61 Ainsi, même si l'absence de certains membres de la famille assure la permanence des familles dans les lieux d'origine, ce maintien n'est possible que par la présence de ces femmes qui se dotent de rôles et d'activités diverses au coût social parfois très élevé. La mobilité, quelle que soit la forme qu'elle revêt, ne peut s'inviter au sein des familles par le départ de certains de ses membres que parce que d'autres peuvent rester.

\section{Éléments de conclusion}

62 Au Nicaragua, dans la vallée de Rio Negro, partir en migration implique que certains restent dans le lieu d'origine. Et ce sont notamment les femmes qui restent. "Rester ", lorsque la mobilité s'établit dans les lieux d'origine implique, même temporairement, des changements dans l'organisation familiale. Les femmes jouent alors un rôle déterminant dans le maintien et le fonctionnement de ces «foyers mobiles» dont la cohésion est sans cesse mise à l'épreuve par des facteurs externes et internes. L'approche en termes de système familial muli-localisé permet alors de saisir le positionnement des femmes au sein des logiques de dispersion et de circulation des familles, et ce au regard des activités qu'elles peuvent/veulent ou non développer. Nos observations rendent compte d'un enchevêtrement et d'une interdépendance des formes de mobilités portées par les différents membres de la famille et des effets de distance complexes (physique, représentation) qui leur sont associées, mais également des circulations matérielles parfois converties en ressources locales lorsque la dispersion entretient des liens et des interactions intrafamiliales. Au cœur de ces dispositifs socio-spatiaux, prenant des configurations spécifiques et mouvantes pour chaque famille, les femmes «qui restent » apparaissent comme des actrices centrales des processus mobilitaires. En effet, elles permettent à d'autres de partir tout en maintenant un certain niveau de cohésion sociale au sein de la famille, gérant les activités et les tensions sociales à l'œuvre dans le lieu d'origine. De plus, elles catalysent également des ressources, celles tirées de la migration des autres et de leurs propres mobilités, qu'elles mettent au service de la satisfaction des besoins quotidiens ou de projets d'investissement. Maintenir une présence dans l'espace local les conduit parfois à interrompre leur propre mobilité au départ d'un conjoint ou enfant mais également à mettre en œuvre des mobilités de proximité et de courte durée afin d'assurer le 
quotidien, face aux risques que comportent notamment les mobilités de longues distance et durée. Les modalités de ces formes du rester sont conditionnées à la fois par l'âge, le cycle de vie et leur place dans la famille mais également par les dotations en ressources des familles.

Rester n'est donc en rien synonyme de passivité, ni de fixité. Rester, c'est gérer la mobilité des autres membres de la famille, défier l'absence et ses affronts pour faire présence au quotidien dans l'espace local.

\section{BIBLIOGRAPHY}

Baumeister E., Fernàndez E., Acuña G. 2008. Estudio sobre las migraciones regionales de los nicaragüenses. Guatemala, Editorial de Ciencias Sociales, $103 \mathrm{p}$.

Bertaux D., 2010. L'enquête et ses méthodes : Le récit de vie. Armand Colin. Paris, $3^{\mathrm{e}}$ éd., 126 p.

Cortes G., 2004. Una ruralidad de la ausencia. Dinámicas migratorias internacionales en los valles interandinos de Bolivia en un contexto de crisis. In Hinojosa Gordonava A., Migraciones transnacionales, Visiones de Norte y Sudamérica. La Paz (Bolivia), Cef/Plural Editores, p. 167-199.

Di Méo G., 1985. Les formations socio-spatiales ou la dimension infra-régionale en géographie. Annales de Géographie, 94, n5 526, p. 661-689.

Di Méo G., 1987. Objectivation et représentation des formations socio-spatiales : de l'acteur au territoire. Annales de Géographie, 96, n537, p. 564-594.

Fauné M.-A., 1995. Las familias, las mujeres: qué dice la realidad. Envio, 160.

Fernández Poncela A., 1999. Arreglos y desarreglos familiares (Centroamérica y Nicaragua). Revista Chilena de Antropología, 15, p. 131-144.

Fréguin-Gresh S., Trousselle A., Cortes G., Sourisseau J.-M., Guétat-Bernard H., 2015. Le système familial multilocalisé. Proposition analytique et méthodologique pour interroger les liens entre migrations et développement rural au Sud. Mondes en Développement, 43, 172, 2015/4, p. 1-20.

Glick Schiller N., Basch L., Blanc-Szanton C., 1992. Towards a Transnational Perspective on Migration, Race, Class, Ethnicity, and Nationalism Reconsidered. The New York Academy of Sciences. New York, $276 \mathrm{p}$.

Jimenez J., 2015. El Código de la Familia es el último eslabón de un proyecto de control social. Envio, 398.

Kanaiaupuni, S.-M., 1995. The Role of Women in the Social Process of Migration: Household Organizational Strategies of Mexican Families. Thèse de Doctorat, University of Chicago, Department of Sociology.

Levitt, P., 1998. Social Remittances: Migration Driven Local-Level Forms of Cultural Diffusion. International Migration Review, 32, 4, p. 926-948. 
Ma Mung E., Kamel Doraï M., Hily M.-A., Loyer F., 1998. La circulation migratoire. Bilan des travaux. Synthèse. Migrations études, revue de synthèse sur l'immigration et la présence étrangère en France, $\mathrm{n}^{\circ} 84, \mathrm{p} .1-12$.

Mahler S.-J., 1999. Engendering Transnational Migration: A Case Study of Salvadorans. American Behavioral Scientist, 42, p. 690-719.

Maldidier C., Marchetti R. 1996. El campesino-finquero y el potencial económico del campesinado nicaragüense. Managua, Instituto Nitlapán, $174 \mathrm{p}$.

Oso L., C. Catarino, 1997. Femmes chefs de ménage et migration. In Bisilliat J. (dir.), Femmes du Sud, chefs de famille, Paris, Karthala, p. 73-77.

Organizacion Internacional para las Migraciones, 2013. Perfil migratorio de Nicaragua 2012. Managua, $211 \mathrm{p}$.

Parreñas R., 2005. Children of Global Migration. Transnational Families and Gendered Woes. Stanford, Stanford University Press.

Pessar P., 2005. Women, gender, and international migration across and beyond the Americas: Inequalities and limited empowerment. Communication présentée à la conférence Expert group meeting on international migration and development in Latin America and the Caribbean, Mexico City.

Poinard M., 1991. Les portugais dans l'émigration: une géographie de l'absence. Thèse de Doctorat, Université de Toulouse 2.

Prunier D., 2013. De nouvelles ruralités en Amérique centrale ? Dynamiques de mobilité, ressources et organisations familiales. Thèse de Doctorat de Géographie, Université Paris Diderot - Paris 7, 471 p.

Quesnel A., Del Rey A., 2005. Mobilité, absence de longue durée et relations intergénérationnelles en milieu rural (État du Veracruz, Mexique). Cahiers des Amériques latines, nº 45, p. 75-91.

Taylor M., Moran-Taylor M.-J., Ruiz D.-R., 2006. Land, ethnic, and gender change: transnational migration and its effects on Guatemalan lives and landscapes. Geoforum, 37, p. 41-61.

Trousselle A., 2012. Les mobilités rurales au Nicaragua, au prisme du transnationalisme et des modèles d'archipel familiaux : caractéristiques et perspectives. Mémoire de fin d'études ISTOM, Cergy, $116 \mathrm{p}$. Vermot C., 2015. Capturer une émotion qui ne s'énonce pas. Terrains/Théories, $\mathrm{n}^{\circ} 2$.

Winters N., 2014. Responsibility, Mobility, and Power: Translocal Carework Negotiations of Nicaraguan Families. International Migration Review, 48, 2, p. 415-441.

\section{NOTES}

1. Nous entendons par " celles qui restent ", les femmes résidant dans leur lieu d'origine (au sein de la zone d'étude) directement concernées par le départ en mobilité (d'une durée égale ou supérieure à 3 mois) d'un proche, homme ou femme, avec qui elles partageaient jusqu'au départ le même foyer. Il peut alors s'agir du départ de leur conjoint, de leurs enfants, voire dans quelques cas de leur frère ou sœur à condition que les types de liens maintenus témoignent d'une organisation collective (maintien de l'appartenance au foyer devenu «mobile»). Dans cette situation, les femmes restent dans leur lieu d'origine et ne recourent éventuellement qu'à des mobilités de proximité (locales, régionales) et/ou qui impliquent de courtes absences.

2. Nous définissons le « lieu d'origine» comme le lieu (la communauté ou le bourg rural) auquel la personne se sent appartenir. Il s'agit généralement du lieu où la première génération de la famille est née ou s'est installée, et où les générations descendantes sont nées et ont résidé. 
3. Nous distinguons deux catégories de mobilités, celles qui impliquent un changement de résidence sur une durée de plus de trois mois (migration) et les mobilités non résidentielles qui n'impliquent donc pas de changement de résidence. Les mobilités résidentielles correspondent principalement à des mobilités de travail mais traduisent également des situations de regroupement familial. Elles renvoient également à des volontés de changer de lieu de vie, dans la majorité des cas afin d'accéder à de meilleures ressources pour la réalisation d'activités agricoles et non agricoles. Ces mobilités résidentielles se font en direction des pays d'Amérique centrale, vers les États-Unis et l'Espagne mais aussi à l'échelle nationale (Somotillo, Chinandega, San Carlos).

Les mobilités non résidentielles renvoient à des mobilités aux temporalités variées, elles sont quotidiennes, hebdomadaires ou mensuelles et liées à la commercialisation de la production ou de produits transformés et manufacturés, à la réalisation d'études supérieures. Ces mobilités se réalisent vers les pôles urbains de la région d'étude, du département de Chinandega, des espaces frontaliers du Honduras mais également vers des communautés rurales de la région d'étude.

4. Les différentes collectes de données ont été réalisées en 2012 (6 mois), en 2013 (2 mois) dans le cadre de stages de fin d'études ainsi qu'en 2014 ( 1 an) et en 2016 (3 mois) dans le cadre d'une recherche doctorale.

5. L'arrivée des Sandinistes au pouvoir constitue un tournant politique important avec la confiscation des terres latifundiaires et la redistribution sous la forme de coopératives. Cette situation est couplée à une guerre civile menée par les «Contras » appuyés par les États-Unis qui se déploient notamment le long de la frontière qui borde la vallée du Rio Negro. Dans le même temps, le pays est confronté à un blocus économique (1985).

6. La décennie 1990 correspond à une phase de libéralisation de l'économie avec notamment le démantèlement des coopératives agricoles et l'instauration de la propriété privée. Les Accords de Paix sont signés en 1992.

7. En 2006 est signé le Convenio Centroamericano de libre movilidad (CA-4) entre le Guatemala, le Salvador, le Honduras et le Nicaragua qui permet aux individus de circuler au sein de ces pays uniquement munis de leur carte d'identité.

8. Ce terme s'inspire du concept de «formation socio-spatiale» de Guy Di Méo. Nous tentons notamment d'identifier des formations sociales propres, celles familiales, productrices d'un espace, de par les liens sociaux maintenus, dont la couverture spatiale est discontinue (Di Méo, 1985, 1987).

9. Si nous nous focalisons sur les femmes «qui restent ", certains hommes « restent » également lorsque les femmes (au sens de conjointe ou fille résidant, jusqu'à leur départ, dans le même foyer) ou leurs fils partent.

10. Parmi les 283 individus de l'échantillon (enquêtés directement ou renseignés via un entretien mené auprès d'un proche), 30 individus (de plus de 15 ans) originaires de la zone d'étude ont été directement enquêtés dans certains lieux de destination (Nicaragua, Costa Rica, Espagne, ÉtatsUnis).

11. Le suivi mensuel a été mis en place auprès de 6 foyers sélectionnés en fonction de la diversité des activités pratiquées localement et/ou de l'existence de formes de mobilités représentatives au sein de la zone d'étude. Le lien de confiance et la qualité de la relation entretenue avec les enquêtés a également constitué un critère de sélection de ces foyers.

12. Nous avons sélectionné des familles nucléaires de référence dans les différents milieux agroécologiques de la zone d'étude, en veillant à prendre en compte une diversité de situations en termes de cycle de vie de la famille, d'activités réalisées localement et de mobilités mises en œuvre. Notre démarche a été d'enquêter, dans la mesure du possible, la totalité des membres de la famille élargie (de plus de 15 ans), quelle que soit leur localisation.

13. Nous définissons la famille ainsi : un couple fondateur qui constitue la première génération à partir duquel se déploie sa descendance basée sur les liens de parenté, les alliances (mariage, 
concubinage, couples officieux) ou d'autres liens de proximité sociale comme l'adoption informelle. La famille a été étudiée sur trois générations (ce qui n'exclut pas que la $4^{\mathrm{e}}$ génération ait été recensée dans certaines familles). Les groupes familiaux correspondent alors aux fratries de la deuxième génération ainsi qu'à leurs descendances. Enfin, le «foyer » correspond à la famille nucléaire (parents et enfants) résidant ou non sous un même toit.

14. En comparaison, les hommes représentent $52 \%$ de notre échantillon soit 147 individus. Au moment des enquêtes, $57 \%$ ont au moins une expérience passée ou actuelle de mobilité, $25 \%$ sont en mobilité résidentielle et $6 \%$ pratiquent des mobilités non résidentielles. Ces dernières correspondent uniquement à des migrations saisonnières de 3 mois maximum contrairement à celles mises en œuvre par les femmes qui renvoient à différentes formes de mobilités de proximité et de courte durée. Au final, seulement 14 hommes ( $9,5 \%$ de l'échantillon) sont considérés comme des hommes « qui restent ». Parmi ces 147 hommes, 97 résident dans leur lieu d'origine (66\%). 1 d'entre eux est devenu un homme « qui reste » suite au départ de sa conjointe et 13 suite au départ d'un fils, ce qui fait un total de 14 hommes « qui restent ».

15. De même, il existe également des situations où la seconde génération se charge d'apporter les soins aux personnes âgées (génération 1) qu'ils prennent sous leur toit. Quoique peu fréquente dans notre échantillon ( 2 cas sur 31 femmes "qui restent»), ces situations témoignent de la pluralité des rôles tenus par ces femmes.

16. Afin de respecter l'anonymat des enquêtés, les prénoms ont été modifiés.

17. Au Nicaragua, les 15 ans sont l'âge clé pour les jeunes filles. Leur fille aura 15 ans en 2018, cela témoigne de la représentation qu'a Clara de leur situation et de leur niveau d'endettement.

18. Parmi les "femmes qui restent», 4 réalisent des activités commerciales impliquant des mobilités à l'échelle régionale. Ce type d'activité est assez commun dans la zone d'étude mais concerne des individus dotés d'un certain niveau de ressources (capacité à financer les déplacements et le transport des marchandises notamment).

19. 10 d'entre elles ont des expériences passées de mobilité résidentielles, et pour 7 d'entre elles, cette expérience renvoie à un changement de résidence au sein de la zone d'étude lors de leur mise en couple.

20. Tout projet mobilitaire possède une part d'improvisation et vise des objectifs parfois flous. Nous le caractérisons néanmoins par les critères suivants: un lieu de destination, une durée (estimée), une activité exercée, un objectif/motivation (plus ou moins clair donc).

21. Les circulations évoquées sont principalement matérielles et correspondent majoritairement à de l'argent. Circulent également des biens consommables (aliments, vêtements, médicaments, etc.) et du matériel et des intrants agricoles.

\section{ABSTRACTS}

This article aims to examine the role of the women "who stay" in the place of origin when other family members go on migration. Applied to a rural area of Nicaragua, the results show that these women have specific roles and activities in the absence of their husbands or children. These situations often lead them to develop forms of immobility or also forms of mobilities which are interesting to analyze in relation to other forms of mobility produced by family members. We propose a typology of these forms "of staying" while analyzing the realities of absence. The objective is to understand the terms of the presence of these families in this rural area. 
Cet article interroge la situation des femmes «qui restent » dans le lieu d'origine, à savoir une région rurale du Nicaragua, lorsque d'autres membres de la famille partent en mobilité. Les résultats montrent que ces femmes exercent des rôles et des activités particuliers en l'absence des conjoints ou des enfants. Ces situations les conduisent notamment à développer des formes d'immobilités ou alors de mobilités de courtes distances et durées qu'il est intéressant d'analyser au regard des autres formes de mobilités produites par les membres de la famille. Nous proposons alors une typologie de ces formes du rester tout en rendant compte des réalités véhiculées par l'absence. Ce afin de saisir les modalités de la présence de ces familles dans cet espace rural.

\section{INDEX}

Mots-clés: circulation, famille, forme de mobilité, multi-localisation, Nicaragua

Keywords: circulation, family, form of mobility, multilocalization, Nicaragua

Subjects: Sur le Champ - Sur le Terrain

\section{AUTHOR}

\section{ANAÏS TROUSSELLE}

Anaïs Trousselle, anais.trousselle@univ-montp3.fr, Doctorante en Géographie à l'Université PaulValéry (Montpellier III) est membre de l'UMR ART-Dev (5281) et membre associée du Laboratoire Mixte International « Mobilités, Gouvernance et Ressources dans le bassin méso-américain » (LMI MESO). Elle a publié récemment :

- Fréguin-Gresh S., Trousselle A., Cortes G., Sourisseau J-M., Guétat-Bernard H., 2015. Le système familial multilocalisé. Proposition analytique et méthodologique pour interroger les liens entre migrations et développement rural au Sud. Mondes en Développement, 43, 172, 2015/4, p. 1-20.

- Fréguin-Gresh S., Trousselle A., Cortes G., 2015. L'agriculture familiale diversifiée multilocalisée au Nicaragua. In Bosc P.-M., Sourisseau J.-M., Bonnal P., Gasselin P., Valette É., Bélières J.-F. Diversité des agricultures familiales : Exister, se transformer, devenir. Versailles, Ed. Quae, coll. Nature et société, p. 95-110. 